\title{
Bayesian Hierarchical Models for Measuring Varietal Improvement in Tobacco Yield and Quality
}

\author{
A. Ford Ramsey ${ }^{1 \star(D)}$ and Roderick M. Rejesus ${ }^{2}$ \\ ${ }^{1}$ Department of Agricultural and Applied Economics, Virginia Tech, Blacksburg, VA, USA and ${ }^{2}$ Department of Agricultural \\ and Resource Economics, North Carolina State University, Raleigh, NC, USA \\ ${ }^{*}$ Corresponding author. Email: aframsey@vt.edu
}

\begin{abstract}
We measure the economic impact of varietal improvement and technological change in flue-cured tobacco across quantity (e.g., yield) and quality dimensions under a voluntary quality constraint. Since 1961, fluecured tobacco breeders in the United States have been subject to the Minimum Standards Program that sets limits on acceptable quality characteristics for commercial tobacco varieties. We implement a Bayesian hierarchical model to measure the contribution of breeding efforts to changes in tobacco yields and quality between 1954 and 2017. The Bayesian model addresses limited data for varieties in the trials and allows easy generation of the necessary parameters of economic interest.
\end{abstract}

Key words: Bayesian hierarchical model; crop quality; genetic improvement; technological change; tobacco; variety trials JEL classifications: Q16; C11; D24

\section{Introduction}

Technological change through plant breeding and improved management practices has enhanced farm productivity and increased agricultural output. The literature on varietal improvement is well-established and focuses on the attribution of changes in yields to different sources, including plant breeding advancements that result in varietal yield improvements (Babcock and Foster, 1991; Nalley et al., 2008, 2010; Nolan and Santos, 2012; Shi et al., 2013b; Wang et al., 2021). Several studies have also recognized the important role of yield risk and empirical linkages between risk, changes in production technologies, and economic outcomes (Brink and McCarl, 1978; Chavas et al., 2001; Shi et al., 2013a). However, increased yield potential and reduced yield variability are not the only attributes of economic significance derived from breeding-based crop improvement. Breeding efforts may be directed toward other attributes with consequent impacts on the commercial viability of different crop varieties.

Quality is an important additional dimension of technological change and crop improvement. In cases where the price is a function of quality, crop breeders can improve farm revenues by developing higher-yielding varieties, higher-quality varieties, or both. Voon and Edwards (1991) and Voon and Edwards (1992) measure significant research benefits from quality improvements in hogs and wheat, respectively. Nogueira et al. (2015) find significant welfare impacts of yield improvement within different classes of wheat. In general, farmers account for different varietal attributes (including quality attributes) when adopting new varieties (Maligalig et al., 2021). However, the extent to which yield and quality can be simultaneously improved by breeders, or

(C) The Author(s), 2021. Published by Cambridge University Press on behalf of the Southern Agricultural Economics Association. This is an Open Access article, distributed under the terms of the Creative Commons Attribution licence (https://creativecommons.org/licenses/by/4.0/), which permits unrestricted re-use, distribution, and reproduction in any medium, provided the original work is properly cited. 
whether there are tradeoffs when breeding for multiple varietal attributes, determines the overall revenue impacts of varietal improvement and the payoffs from public and private breeding research.

Changes in crop quality can either accentuate or negate returns to changes in crop yield. There may be points of diminishing marginal breeding gains where further gains in yield cannot be attained while maintaining or enhancing other varietal attributes valued by consumers. For instance, Nalley et al. (2018) find that relaxation of quality standards in wheat breeding in South Africa would have resulted in gains to yield of around 10-20\%. Their findings have significant economic implications for South Africa's breeding program, as well as their wheat quality regulations.

The objective of this study is to determine the contribution of plant breeding and other technological innovations to changes in both yield and quality of flue-cured tobacco in the United States (U.S.). We do so through a Bayesian hierarchical model that has some advantages in the variety trial setting. These advantages are not unique to tobacco but rather follow from the nature of variety trial data, and models of this type can be used for other crops. As discussed in more detail below, variety trial data often constitute an unbalanced panel with many groups or clusters. The Bayesian approach enables more reliable inference about these groups and their associated parameters.

Our focus is on tobacco because of a unique voluntary agreement affecting the ability of U.S. tobacco breeders to change tobacco quality. Flue-cured tobacco breeders have adhered to this voluntary constraint on quality since 1963 . There is a concern that breeders adhering to the quality standards have adversely influenced the extent of yield gains derived from conventional breeding. That is, whether the rules that require breeders to meet tobacco quality constraints have limited the observed varietal improvements in yields over time. Our empirical setting provides a unique opportunity to examine whether public and private investments in tobacco breeding have resulted in improved yields, even in the presence of a regulatory structure that requires them to maintain quality standards. Findings from this investigation have important implications for the design of regulations surrounding plant innovation and the future of tobacco breeding.

A growing subset of the literature in production economics has explicitly recognized the importance of crop quality as an additional dimension of agricultural products (Kawasaki, 2018; Kawasaki and Lichtenberg, 2013; Kawasaki and Uchida, 2016; Ramsey et al., 2021; Shew et al., 2018). Quality may include such characteristics as timing and method of sale, but quality in agriculture is usually related to physical attributes. For instance, crop grade may depend on color, size, or other sensory characteristics. These attributes are important to consumers and end-users who often pay a price premium for high-quality goods. Hedonic studies have demonstrated the economic importance of quality in pricing for a wide variety of commodities (Donnet et al., 2010; Espinosa and Goodwin, 1991; Golan and Shalit, 1993; Hussein and Fraser, 2018; Stiegert and Blanc, 1997).

Several authors have also investigated trends in tobacco yields and quality over time using field trial data. Bowman et al. (1984) and Babcock and Foster (1991) measured the contribution of breeding to flue-cured tobacco yield gains. Bowman et al. (1984) also considered gains in quality as measured by the percentage of nicotine and total nitrogen in the cured leaf. These authors generally found that genetic improvements contributed to yield gains, but that the effect of technological change (other than breeding advances) was larger. Our study contributes to the literature in several dimensions. First, with the large number of named varieties in their trial data sets, Babcock and Foster (1991) and Bowman et al. (1984) established aggregate varietal groups to ensure tractable estimation. In particular, groups of new and old varieties were established (or compared to a single check variety) and differences in linear trends between varietal groups were used to measure breeding contributions to yield. Varietal effects were estimated for groups (or categories) rather than separately estimating genetic effects for each unique variety. Our study estimates 
variety-specific breeding-based improvements instead of more aggregate "varietal-group" contributions to yield and quality. ${ }^{1}$

Second, both Babcock and Foster (1991) and Bowman et al. (1984) used variety trial data up to the middle 1980s. The year 1982 coincided with the release of the highly successful variety K 326 . Around this time, an apparent plateau in the observed distribution of yields occurred. However, an apparent plateau in the raw data does not necessarily imply a lack of varietal improvement. A plateau conditional on weather, management practices, and the composition of the tobacco varieties in the trials provides stronger evidence of a lack of varietal improvement. Our study is the first to consider variety-specific contributions to both quality and quantity, using more sophisticated and scientifically motivated controls for weather, and using a Bayesian methodology that accommodates the inherent shortcomings of variety trial data.

Lastly, we introduce and implement a Bayesian multilevel model that is well-suited for dealing with unique features of variety trial data sets widely utilized in the agricultural economics literature. The Bayesian framework has several advantages in this setting. First, uncertainty around functions of parameters is relatively easy to compute; credible sets and inferential procedures are straightforward when measuring breeding contributions to yield and quality. Second, the Bayesian model deals well with group variables with a limited numbers of observations by borrowing information from other groups when estimating parameters for the groups of limited size. This is especially important in variety trials where certain crop varieties (i.e., the groups) are only tested in 1 or 2 years. Third, the use of weakly informative priors can aid estimation, especially in the case of many so-called nuisance parameters. Even though the Bayesian multilevel model is applied for flue-cured tobacco varietal trials in this study, the same framework can be applied in a number of economic contexts (e.g., when using other field trial data or other data types with richer variety and/or genetic trait information). It is also flexible as it can be extended to incorporate additional nonlinearities or covariates at the group levels.

Using data from the North Carolina official variety trials (OVTs) from 1954 to 2017, which include measurements of flue-cured tobacco yield and cured leaf chemical analysis, our findings suggest that the Minimum Standards Program has been effective at maintaining the quality standards of new tobacco varieties developed by breeders, and this quality constraint has not precluded further yield improvements from conventional tobacco breeding. This implies that tobacco breeding has not reached a yield plateau even with current quality standards.

\section{Quality Constraints in U.S. Tobacco}

Tobacco is one of the oldest crops cultivated in the U.S.; it was planted and smoked by Native Americans prior to the arrival of Europeans. Finding growing conditions in Virginia to be advantageous for tobacco production, early British colonists produced large quantities of the crop (Hawks and Collins, 1993). Tobacco production became so endemic that tobacco leaf was the only legal currency at times. Tobacco was a fixture in early U.S. agricultural policy with prices stabilized under New Deal agricultural legislation.

Tobacco in the U.S. can be distinguished by federally designated classes and types; the former is largely defined by curing method and the latter by leaf characteristics or geographical indicators. The federal grading system was important in the development of tobacco auction markets as it allowed for efficient marketing under supply controls. With the advent of contracted production and the end of the tobacco quota program in 2004, the grade system is of less practical use today (Dimitri, 2003). The major classes of tobacco are flue-cured, fire-cured, air-cured, dark air-cured,

\footnotetext{
${ }^{1}$ Moreover, in Babcock and Foster (1991) and Bowman et al. (1984), strict assumptions were made on yield trends and only precipitation across the early part of the growing season was controlled. We use more flexible trend and weather specifications. Moreover, in Babcock and Foster (1991) and Bowman et al. (1984), strict assumptions were made on yield trends and only precipitation across the early part of the growing season was controlled. We use more flexible trend and weather specifications.
} 
cigar-filler, cigar-binder, cigar wrapper, and others. Flue-cured tobacco accounts for the largest portion of tobacco receipts in the U.S. (currently as well as historically) and is the class of tobacco we consider in this study. Revenue from flue-cured tobacco depends on both the quantity produced as well as quality. Flue-cured tobacco quality is defined by several factors. From the point of view of the tobacco buyer, the quality of an individual lot is also related to the way that the leaf will be blended before the production of the final product. The main end-use for flue-cured tobacco is in cigarettes, where it is blended with burley and oriental tobacco. Therefore, desirable traits in flue-cured tobacco leaf are directly related to the suitability of the tobacco for use in cigarettes. Hawks and Collins (1993) divide the quality attributes of tobacco into physical and chemical properties. Among important chemical properties are nicotine, sugars, and nitrogen, which can be precisely measured, and for which minimum acceptable standards have been established.

Several attempts have been made at devising quality indices based on the chemical properties of tobacco leaf. Some examples by Pyriki (1958) and Bruckner (1936) are reported in Tso (1972). Quality indices have generally included total alkaloids (of which nicotine is the major part) as well as reducing sugars. Hawks and Collins (1993) suggest that sugar content can be positively associated with tobacco quality but only within a certain range. Too high or too low a sugar content impairs the quality of the leaf and consumer acceptability. Sugar content cannot be considered alone but must be considered in relation to alkaloid content.

The primary alkaloid in flue-cured tobacco is nicotine. As shown in Tso (1972), tobacco which has too high or too low a ratio of sugars to alkaloids is either too harsh in flavor and highly irritating, or too mild. The highest quality tobacco will have a ratio of sugars to alkaloids that is within a defined range. Weybrew et al. (1983) suggest that the optimal ratio of reducing sugars to nicotine is in the range of 6-9 with Maw et al. (2009) implying a preferred ratio of 10 . The ratio of sugars to alkaloids is the quality measure we use in our empirical analysis.

Quality aspects of tobacco leaf are necessary to understand the historical development of tobacco markets in the U.S., particularly standards for new varieties and public/private breeding programs. The Agricultural Adjustment Act of 1933 established tobacco as a basic crop with the intention of stabilizing tobacco prices. Early in tobacco program history, price supports were accomplished through an acreage restriction. Each year, the Secretary of Agriculture would set the total amount of tobacco acreage, and this number would be apportioned among the states, counties, and individual producers. The tobacco program would later move to a poundage quota and was eventually eliminated, the economics of which resulted in several studies (Brown et al., 2007; Rucker et al., 1995). With an acreage allotment program, limits on the amount of land in production are thought to have caused production intensification. It has also been suggested that the acreage quota encouraged breeding of varieties that were exceptionally high yielding but of poor quality. Concerns about the quality of new varieties of U.S. tobacco, resulting from the acreage allotments of 1939, began to grow in the late 1950s. Three varieties (Coker 139, Coker 140, and Dixie Bright 244) were released in 1954 and 1955. By 1956, at least half of the acreage in North Carolina was planted to Coker 139 (Bowman, 1996). These varieties had excellent yield potential but at the expense of quality. They had high sugar content and low total alkaloid content, especially nicotine. In the OVT dataset used in this study, 6 of the 10 largest sugars to alkaloid ratios are observed for varieties Coker 139 or Coker 140. These observations have sugar to alkaloid ratios over 20 in comparison to the optimal ratio of $6-10$.

While tobacco manufacturers could refuse to purchase low-quality tobacco, the federal government was obligated to make purchases through the Commodity Stabilization Service. Such large quantities were placed with the Commodity Stabilization Service that, beginning in 1957, the price support for these three specific varieties was considerably reduced. This required government workers to be able to identify the variety being grown to ensure that the tobacco was discounted when marketed. Concern was so great that any farmer reporting a discounted variety grown on his 
farm would have all tobacco from his farm, regardless of variety, considered discount tobacco. Additional varieties would be discounted throughout the late 1950s and early 1960s and later termed "discount varieties" or "barn-buster varieties." Price differences between discount varieties and acceptable varieties could be large. For much of the life of the tobacco program, discount varieties eligible for price support received a price roughly $50 \%$ less than standard varieties; clearly a significant discount with major impacts on farm revenues. The response of producers to the acreage allotment appears to be a textbook case of market intervention, perverse incentives, and unintended consequences.

Recognizing the increasing popularity of low quality, high yielding varieties, the tobacco industry collectively worked together to institute a program that would control the quality of varieties being released to the market. The first formal attempt at setting quality standards came at the 1958 Tobacco Workers' Conference where a Regional Flue-Cured Tobacco Quality Evaluation Committee was created. The Tobacco Workers' Conference is a gathering of those involved in U.S. tobacco including private breeders, public breeders, academics, and tobacco industry representatives. It was not until the 1961 Tobacco Workers' Conference that minimum release standards were approved by a subcommittee on Tobacco Varietal Release Standards. The Minimum Standards Program came into force in 1963. As noted in Bowman (1996), the initial standards specified that leaf should be analyzed for nicotine, soluble sugars, and nitrogen, leaf should be compared to the proposed check varieties Hicks Broadleaf and Vesta 5, smoke results would be obtained from the tobacco industry, and the tobacco industry would accept the leaf for commercial release.

After 1963, the official check varieties were Hicks Broadleaf and NC 95 and new varieties were mandated to be plus or minus 15\% from the total alkaloids and soluble sugars (among other chemicals) averaged over the checks. Starting in 1967, the total alkaloid standard was adjusted to plus $15 \%$ and minus $20 \%$ of the check varieties due to concerns with increasing nicotine levels. Given that the primary alkaloid in tobacco is nicotine, the requirements on total alkaloids were changed to a standard on nicotine in 1970. NC 2326 took the place of Hicks Broadleaf as a check in 1969 and was eventually replaced by K 326. Regardless of minor changes over time, the substantive quality effects of the Minimum Standards Program have remained the same since its inception.

There is evidence that the standards program, in spite of its voluntary nature, has helped maintain quality metrics to a standard acceptable to manufacturers. Bowman (1996) notes that all varieties released under the Minimum Standards Program have continued to be made available for commercial use and none were retroactively discounted. The voluntary minimum standards are likely successful because, in spite of any official enforcement mechanisms, reputation effects may constrain actions of any individual party (Greif, 1993). The tobacco manufacturing and breeding community are concentrated; as early as 1987, there were only four private breeding organizations and four public breeders. By 1996, there were seven tobacco companies involved in standards setting and testing. MacLeod (2007) provides an overview of the many ways that contracts can be enforced in different institutional settings.

\section{Data Description}

Our data are drawn from OVT of flue-cured tobacco conducted by North Carolina State University between 1954 and 2017. North Carolina State University is the only remaining public flue-cured breeding program in the country. The data are an unbalanced panel with yields and quality measured for a total of 306 flue-cured varieties, over 63 years, and in seven locations, resulting in 6,966 variety-location-year observations. Trial locations are shown on a map in 
Figure A1 of the online appendix. The varieties included in the OVT data are varieties for commercial release. No commercial varieties of flue-cured tobacco in the U.S. are transgenic; all genetic modification has been performed through conventional breeding. ${ }^{2}$

Figure A2 of the online appendix shows a histogram of varieties by the number of years present in the OVT data. The histogram is strongly right-skewed with the majority of varieties appearing in the trials less than 5 years. The mean number of years in the sample was 6 with standard deviation of roughly 6 years. In spite of the short lifespan of most varieties, some varieties appear in the data for an extended period of time. These varieties were included in multiple years either because they were official check varieties or simply entered in the trials for comparison.

We use the sugars to total alkaloids ratio as a measure of quality. However, information on sugars and total alkaloids were not collected in every location-year combination. In some years, sugars were defined as reducing sugars while in others they were defined as soluble sugars. These are equivalent definitions referring to the way that the sugar content of a sample is measured. With respect to alkaloids, we observe total alkaloids or various constituent alkaloids, namely nicotine and nornicotine. Although tobacco contains a number of different alkaloidal materials, Tso (1972) suggests that the importance of different alkaloid constituents in commercial tobacco is, ordered from most important to less important: nicotine, nornicotine, anabasine, etc. To construct the measure of total alkaloids when not observed, we summed nicotine and nornicotine content. No cured leaf analysis was available in 1992 and any observations with missing leaf analysis were dropped in the quality model estimation. ${ }^{3}$

Weather variables are also utilized in the study and were constructed from data used in Schlenker and Roberts (2009). This data set is based on PRISM weather data and maps temperatures and precipitation to a 2.5 mile by 2.5 mile grid of the contiguous United States. We mapped the postal address of the experiment stations in the trial data to the corresponding longitude and latitude, and then to the grid cell containing the experiment station. The only location that could not be exactly matched was Rural Hall; this location is only in the data from 1954 to 1961. Archival documents indicated that the trials were conducted on rented land several miles outside of Rural Hall, NC. The weather data for this location are those at the center of the town. The main weather variables included in the analysis are three temperature bins containing degree days inside and outside the optimal growing range for tobacco, as well as total precipitation. All weather variables are measured over the growing season from May 1 to September 1.

Summary statistics for all variables in the regressions are shown in Table 1 . There is variation in yields over time, location, and variety. The minimum yield was observed at Rocky Mount in 2006 (excluding observations where no yield was recorded due to disease). The maximum yield was observed at Rocky Mount in 1981 with the variety Speight G-58. There is also variation in quality as measured by the sugars to alkaloids ratio. The maximum ratio was observed for the discount variety Coker 140 at Whiteville, NC in 1957. Variation in the weather variables suggests that, although all experiment stations are located in North Carolina, there is enough weather variation across space and time to warrant inclusion of observed weather as a control.

Plots of yields and quality ratios over time are shown in Figure 1. In the yield plot (i.e., upper panel), there is an apparent yield plateau in the early 1980s. Furthermore, the Minimum Standards Program does not appear to have prevented yield gains up through the 1970s and 1980s. The plot of the quality ratio indicates that the quality constraints did have the intended effect of driving the ratio down to acceptable levels. However, any information from these plots is only suggestive. The

\footnotetext{
${ }^{2}$ The trials were typically conducted using randomized complete block designs with three replicates per location. The management practices are best management practices based on recommendations from the extension service; management practices do vary by location and year. Management practices are designed to reduce yield reductions due to pests, disease, and other factors. None of the modern trial locations are reported as having significant disease pressure.

${ }^{3}$ The sugar and alkaloid samples were composited (weighted mean) samples collected after curing. These samples represent the entire plant (i.e., all stalk positions).
} 
Table 1. Summary statistics: yield, quality, and weather data

\begin{tabular}{lcccc}
\hline Variable & Mean & Std. Dev. & Min & Max \\
\hline Flue-cured tobacco yield (lbs./ac.) & 2,723 & 497 & 1,009 & 4,634 \\
\hline Sugars (\%) & 16.17 & 4.29 & 1.77 & 29.44 \\
\hline Total alkaloids (\%) & 2.92 & 0.80 & 0.74 & 8.29 \\
\hline Sugars to alkaloids & 6.30 & 3.20 & 0.33 & 26.01 \\
\hline Low degree days & 2,168 & 25 & 2,077 & 2,216 \\
\hline Medium degree days & 781 & 80 & 554 & 1,008 \\
\hline High degree days & 12 & 8 & 1 & 40 \\
\hline Precipitation (mm.) & 484 & 114 & 230 \\
\hline
\end{tabular}

composition of the varieties in the trials changes over time and is not necessarily indicative of yield or quality associated with new varieties. Additionally, weather can cause significant variation in both yield and quality. For instance, 2013 saw low yields and low quality (a high sugars to alkaloids ratio) due to inadequate rain at the beginning of the season and high temperatures. The suggestive evidence in Figure 1 motivates the formal econometric analysis described below.

\section{Empirical Approach}

In both the economic and agronomic literature, varietal improvement can be measured by comparing the mean yield of a variety to the mean yield of a check variety. Comparisons are typically made of mean yield across a single year and several locations. Analysis of variance (ANOVA) is used because these observations are subject to the same weather patterns, soil quality, and management practices. Because we are measuring breeding gains over a long period of time and across many locations, we use a regression-based approach that shares conceptual similarities with analysis of variance (Gelman 2005). If other sources of variation in yield can be accounted for and modeled, and given the chosen regression specification and the randomized trial design, then regression parameters capture the effect of improvements due to breeding, i.e. varietal contributions to both yield and quality.

We use a Bayesian multilevel linear model to estimate differences in group means across varieties and thereby measure improvements in both yield and quality from breeding. Other studies have approached similar empirical situations through the use of frequentist mixed-effects models (Tack et al., 2015). While such models are conceptually similar to a Bayesian multilevel model, the Bayesian paradigm has several advantages. Constructs of parameters can be derived in a straightforward way, and perhaps more importantly, uncertainty surrounding these constructs is just as easily established.

The greatest advantage of a Bayesian multilevel model - in this case-is that such models use all available data to estimate parameters for groups with few members (i.e., some individual varieties in this study). Our goal is to estimate group means for each flue-cured tobacco variety after controlling for weather, location, and other factors. Many varieties included in the trials are observed in the data only for a few years. Some varieties in our dataset are observed only twice (i.e., in one year at two locations). Estimation of group-level means for these varieties, if we were to utilize the limited information for those varieties, would be practically useless in terms of making inferences about genetic contributions of those specific varieties. The Bayesian multilevel model splits the difference between local estimation and a completely pooled regression model; this provides the best estimate of the group means given the uncertainty in the data and the assumptions 

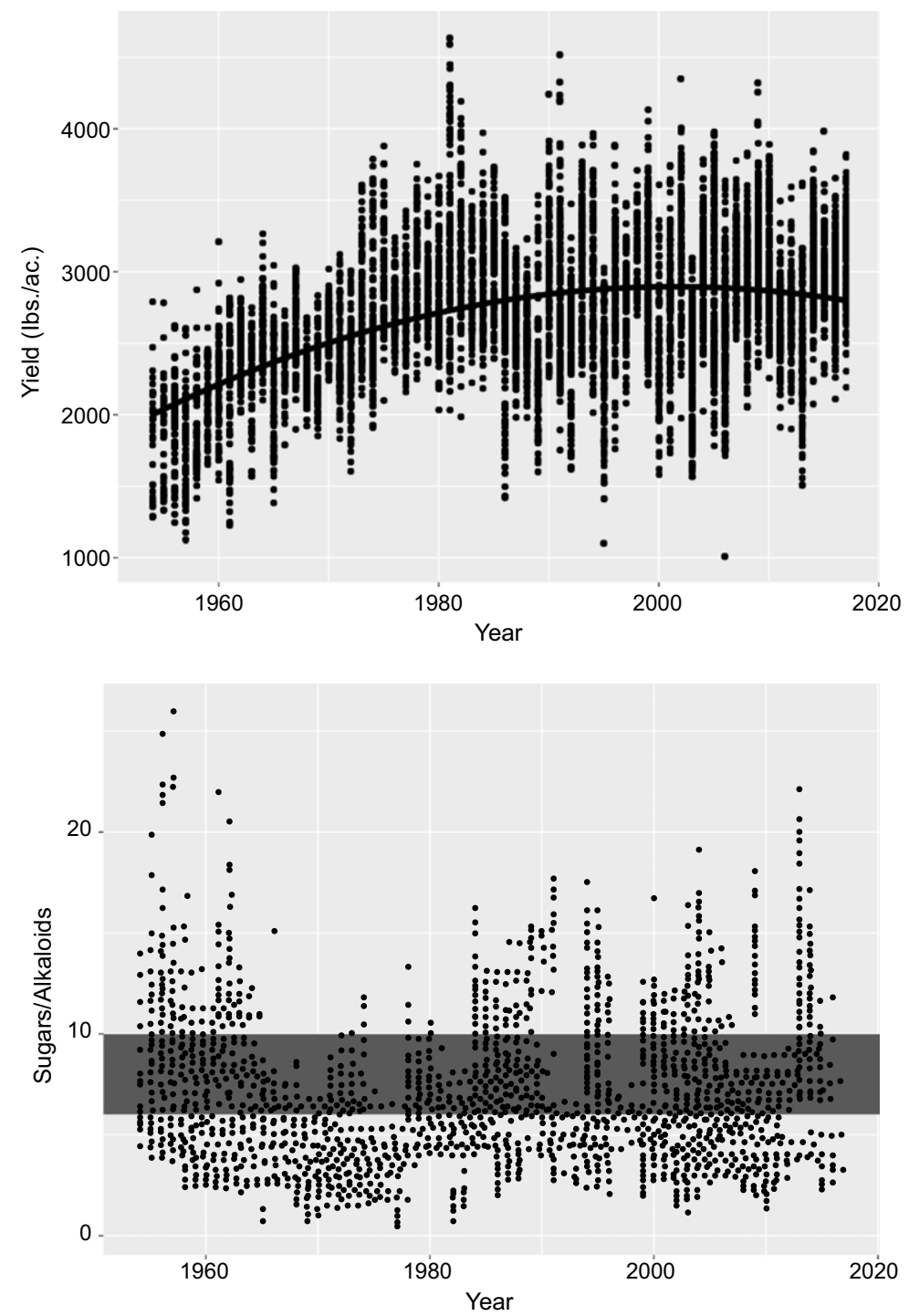

Figure 1. Tobacco Yields and Sugars/Alk. Ratio, 1954-2017.

Note: Dark line is a quadratic trend obtained by regressing yield on year and year squared. Shaded area is suggested ideal range for sugars/alkaloids ratio.

of the model. The multilevel model converges asymptotically to the within-group regressions (Gelman and Hill, 2006).

More formally, we define $y_{i j t}$ as the yield of variety $i$ at location $j$ in year $t$ in pounds per acre. The panel data we use are unbalanced and yields can be econometrically modeled as

$$
y_{i j t} \sim N\left(\mu+\alpha_{i}+\gamma_{j}+\rho_{t}+f\left(X_{j t} ; \beta\right), \sigma_{y}\right)
$$

where $\alpha_{i}$ is the variety-varying intercept, $\gamma_{j}$ is the location-varying intercept, and $\rho_{t}$ is a timevarying intercept. The function $f(\cdot)$ is what Tack et al. (2015) refer to as the climate function. The matrix $X_{j t}$ consists of a variety of weather variables influencing tobacco yields and quality with a vector of coefficients $\beta$. The residual variance is given by $\sigma^{2}$ and $\mu$ is a population-level intercept. 
All parameters in equation 1 are given prior distributions as follows

$$
\begin{gathered}
\mu \sim N(2723,483) \\
\alpha_{i} \sim N\left(0, \sigma_{\alpha}\right) \\
\gamma_{j} \sim N\left(0, \sigma_{\gamma}\right) \\
\rho_{t} \sim N\left(0, \sigma_{\rho}\right) \\
\beta \sim N(0,10) \\
\sigma_{y}^{2} \sim \operatorname{HN}(0,483)
\end{gathered}
$$

where the second parameter of the normal distribution is the standard deviation. The term $H N(\cdot)$ denotes the half-normal distribution. The priors are weakly informative and assumed to be independent; we found that using completely uninformative priors increased computational time but did not have a substantive impact on estimation results. The coefficients in the climate function are all given fairly wide normal priors. The prior for the intercept has a location equal to the mean yield and empirical standard deviation.

There are three group standard deviations that are given hyperpriors. For simplicity, all three standard deviations are given the same prior with

$$
\begin{aligned}
\sigma_{\alpha} & \sim H N(0,483) \\
\sigma_{\gamma} & \sim H N(0,483) \\
\sigma_{\rho} & \sim H N(0,483)
\end{aligned}
$$

resulting again in weakly informative priors.

Similarly, define $s_{i j t}$ as the sugars to alkaloids ratio of variety $i$ at location $j$ in year $t$.

The tobacco quality model can then be specified as

$$
\log \left(s_{i j t}\right) \sim N\left(\mu+\alpha_{i}+\gamma_{j}+\rho_{t}+f\left(X_{j t} ; \beta\right), \sigma_{y}\right)
$$

with the other non-quality variables and parameters defined as in equation 1 . We model quality as the logarithm of the sugars to alkaloids ratio because the underlying data is right-skewed and the ratio can only take positive values. The priors for the quality model are derived in the same way as those for the yield model and again designed to be weakly informative. In particular,

$$
\begin{aligned}
\mu & \sim N(2,10) \\
\alpha_{i} & \sim N\left(0, \sigma^{2}\right) \\
\gamma_{j} & \sim N\left(0, \sigma^{2}\right) \\
\rho_{t} & \sim N\left(0, \sigma^{2}\right) \\
\beta & \sim N(0,10) \\
\sigma_{y}^{2} & \sim H N(0,10) .
\end{aligned}
$$

The priors here are on the scale of the logarithm of the sugars to alkaloids ratio. Note that the models in equations 1 and 2 are separate models each with their own parameters. For simplicity, we do not distinguish between the parameters of the model using any change in notation. We reference either the yield model or the quality model in the empirical analysis that follows.

The hyperpriors for the varying intercepts of the quality model are

$$
\sigma_{\alpha} \sim H N(0,10)
$$




$$
\begin{gathered}
\sigma_{\gamma} \sim \operatorname{HN}(0,10) \\
\sigma_{\rho} \sim \operatorname{HN}(0,10),
\end{gathered}
$$

and again derived as in the yield model.

Although the climate function is not germane to the primary question of economic interest in this study (identifying gains from breeding and technology), it is important to control for observed weather across time and space to accurately identify mean yields across groups, and consequently estimate the variety-varying intercepts. This point has been noted by Tack et al. (2015), Lobell et al. (2011), and Schlenker and Roberts (2006). The climate function is typically specified as a function of temperature and precipitation, and these are measured either across the growing season, by month, or by plant growth stage. An important consideration is that the impact of weather on yields and quality can be nonlinear, and the climate function should be flexible enough to capture nonlinear effects when present.

We specify the climate function as follows:

$$
f\left(X_{j t} ; \beta\right)=\beta_{1} d d_{18}+\beta_{2} d d_{20}+\beta_{3} d d_{32}+\beta_{4} p
$$

which is similar to the specification in Tack et al. (2012) that allows for nonlinearities in the temperature effects. All variables are measured across the growing season for flue-cured tobacco which we assume is from May 1 to September 1 . The variable $d d_{18}$ is the number of degree days above $0^{\circ} \mathrm{C}$ and below $18^{\circ} \mathrm{C}$. The middle category captures degree days between $18^{\circ} \mathrm{C}$ and $32^{\circ} \mathrm{C}$, and the last category captures degree days above $32^{\circ} \mathrm{C}$. Flue-cured tobacco is grown on a variety of soil types and in a variety of climates. The plant is relatively robust to extreme temperatures; Hawks and Collins (1993) suggests that tobacco performs best with daily temperatures between 65 and 90 degrees Fahrenheit (i.e., about 18 to $32^{\circ} \mathrm{C}$ ). This middle range has been suggested as the optimal temperature for tobacco growth by Tso (1972). Precipitation $(P)$ is measured as the total cumulative precipitation over the growing season in millimeters.

We fit the models in equations 1 and 2 using Hamiltonian Monte Carlo implemented through the software Stan. The practical benefit of this computational approach is that it is not necessary to derive conditional posterior distributions of the parameters. ${ }^{4}$ For each model, we ran four separate chains with a warmup period of 2,500 iterations and a sampling period of 2,500 iterations. The draws from the four chains were combined, and inference was conducted on the resulting 10,000 draws. Convergence was assessed using trace plots (shown in the appendix for select parameters), the number of effective samples, and the Gelman-Rubin diagnostic. Both models appeared to converge with favorable values for the diagnostics. Graphical posterior predictive checks in Figure A3 show the actual distribution of the data plotted alongside 10 samples containing replicates from the posterior predictive distribution (Gabry et al., 2019). The replicates are simulated from the posterior predictive distribution with the data used to fit the model. The models do a reasonable job of generating data similar to the data actually observed.

\section{Results and Discussion}

The parameters of interest for measuring contributions of breeding are the varying intercepts associated with a particularly named variety. Figure 2 shows the posterior medians for the variety intercepts plotted against the first year that the variety appeared in the trials. Dotted lines denote the year of first appearance (1982) and variety intercept for K 326.

\footnotetext{
${ }^{4}$ We accessed Stan using the BRMS package developed by Bürkner et al. (2017). More information about Stan can be found at the Stan website: https://mc-stan.org/. Hamiltonian Monte Carlo is, like other sampling algorithms, a means of sampling from a target probability distribution: the posterior in this case. Some details of Hamiltonian Monte Carlo are given in Neal (2011) and Betancourt and Girolami (2013).
} 
(a)

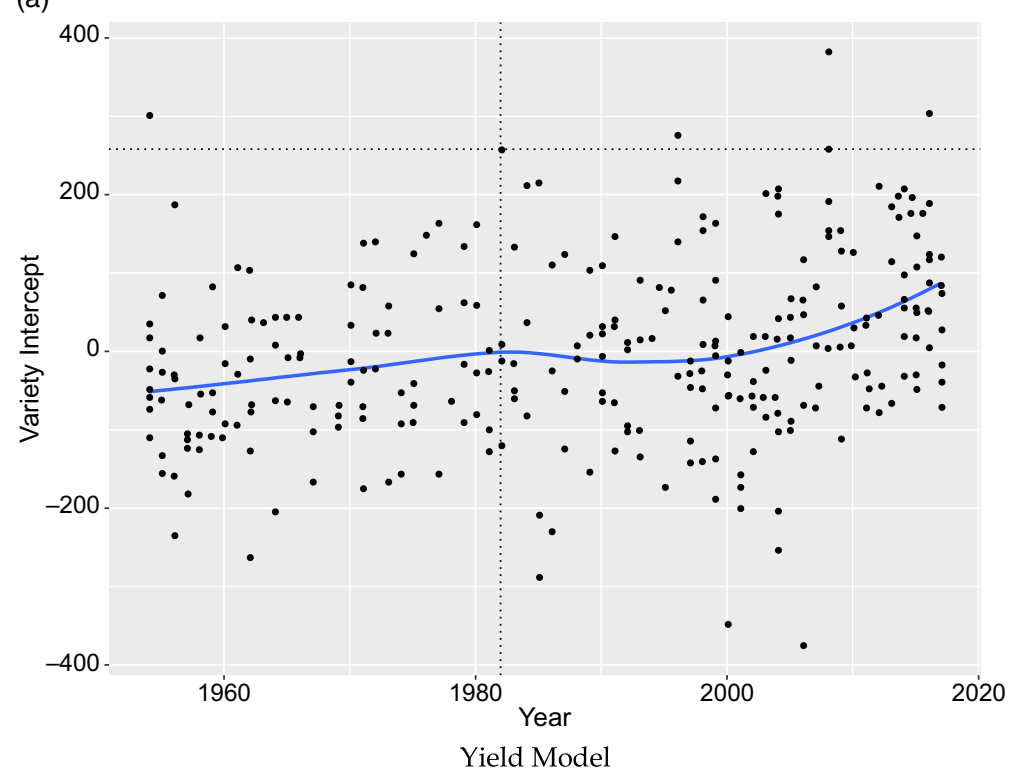

(b)

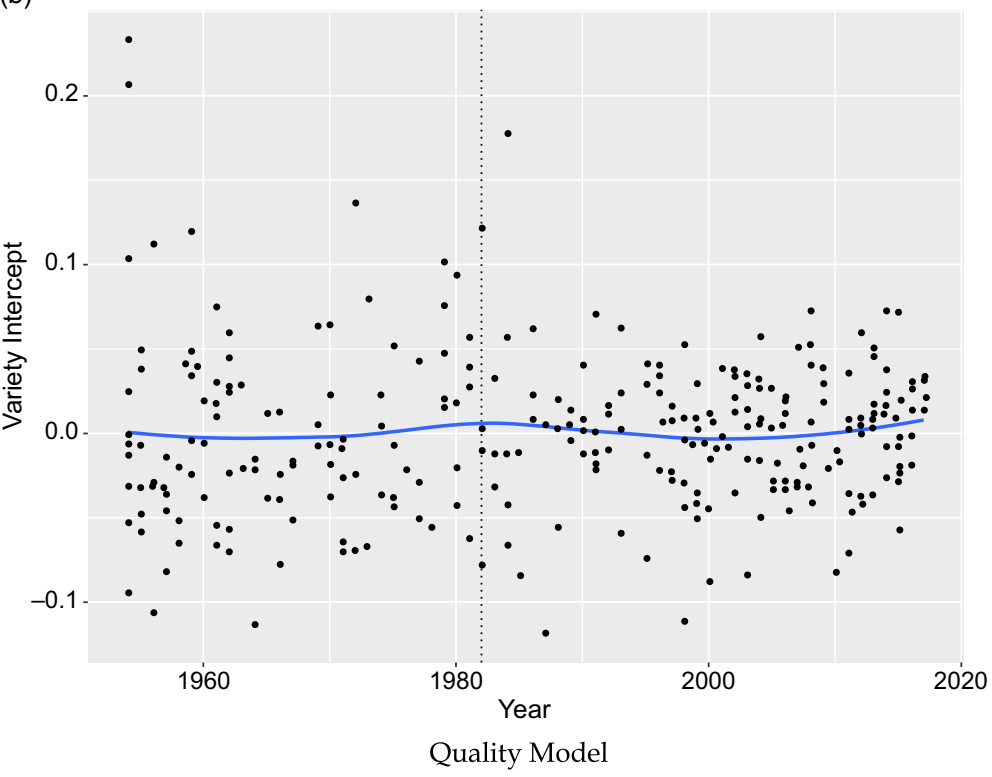

Figure 2. Variety intercepts: variety-specific genetic contributions.

Note: Dotted lines denote 1982 and variety K 326. Variety intercept in the yield model is interpreted as lbs./acre relative to the average yield given by $\mu$. Variety intercept in the quality model is interpreted as sugars/alkaloids ratio relative to the average quality given by $\mu$.

Many of the varieties developed after 1980 were based on K 326 and this variety took a substantial share of the market when released. In terms of breeding contributions to yields, $\mathrm{K} 326$ is special for being particularly high yielding relative to other varieties at the time. A smoothed curve fit the intercepts by locally weighted scatterplot smoothing (LOESS) is also included in the plots.

Based on the variety intercepts and the slope of the LOESS curve, varietal contributions to yields improved until around 1981 at which point yield improvement was stagnant (Figure 2). Only after the year 2000 does the breeding contribution to yields begin to increase again (though 
more rapidly than the pre-1980 period). Three modern varieties are found to be better than $\mathrm{K}$ 326 in terms of yield. On the other hand, there is little evidence that the impact of varietal advances on quality has changed since the introduction of the Minimum Standards Program (e.g., the flat LOESS curve). The program has essentially eliminated tobacco varieties with high yields and high sugar to alkaloid ratios like those observed in the late 1950s and early 1960s. Breeders have followed mandates of the program and their breeding efforts have not significantly altered quality. This is expected because, unlike yields where more is better ceteris paribus, a large proportion of U.S. flue cured tobacco varieties were already of high quality in the 1950s. Any differences in quality are likely the result of changes to management practices or normal weather fluctuations.

We also estimated a piecewise regression for the yield variety intercepts shown in Figure 2. The break point for the piecewise regression was identified using the algorithm of Muggeo (2008). A single break point was identified in 2002. Variety intercepts increased at a rate of 0.76 pounds per acre each year before the break, and 5.20 pounds per acre per year afterwards. Note that few varieties after 2002 have low variety intercepts. The slopes of the regression segments correspond to average increases in breeding contributions to yield, assuming a linear trend within each segment. The significant break in 2002 may correspond to changes in the structure of varietal research and development in tobacco and is consistent with the shape of the LOESS curve.

Figure 3 contains plots of the intercept terms that vary by year. The circles represent posterior medians while the bars give $95 \%$ posterior credible intervals. The time intercepts capture other factors influencing yield or quality that vary by time, but do not vary by location/plot (e.g., location invariant, but time-varying factors). These other factors are not captured by the weather controls or variety intercepts and include changes to management practices and production technology over time. If the distribution of weather and management practices (or input use) in a period is assumed to be constant, then trends in the time intercepts are usually interpreted as technological change. This is clear in the yield model where there is an upward trend from 1954 to 1980 . The changes in the time intercepts are larger than those observed for variety over the same period prior to 1980, which suggests that changes to yields from production technology outpaced breeding gains in the early part of the sample.

The importance of technological change (exclusive of varietal improvement) over the first third of the OVT data is consistent with some specific advances made in tobacco production during that time. The first mechanical harvester was introduced in 1954. Chemical suckering was introduced in the OVT in 1974. Bowman et al. (1984) indicates that increasingly effective herbicides and pesticides were also employed during the 1970s. As well, production technologies were introduced that promoted a better root system for nutrient uptake. All of these technologies had positive impacts on the yields obtained in the trials, especially in the pre-1980s period.

Figure 4 shows the posterior distribution of the variety intercept for yield for four important varieties; the comparison here is similar to a comparison of means between a variety and a check. NC 95 is a check variety introduced in 1961 and used as an early check in the Minimum Standards Program. K 326, as mentioned above, was introduced in 1982 and many subsequent varieties incorporate the features of K 326 . Coker 139 is a discount variety introduced in 1954, and CC 35 is a modern variety introduced in 2008. CC 35 has the highest varietal contribution to yield of all varieties in the sample. Measuring advances due to breeding using these three varieties, there is roughly a 368 pound per acre increase moving from NC 95 to K 326 . The breeding improvements embodied in K 326 contribute less than Coker 139-as measured by the posterior median. However, CC 35 produces roughly 124 pounds per acre over K 326.

Breeding efforts at the frontier can also be measured by the average or maximum genetic contribution of all varieties introduced in that year. These quantities can easily be constructed from the parameters of the fitted Bayesian model. For instance, the average genetic contribution in the year of introduction $t$ would be given by 

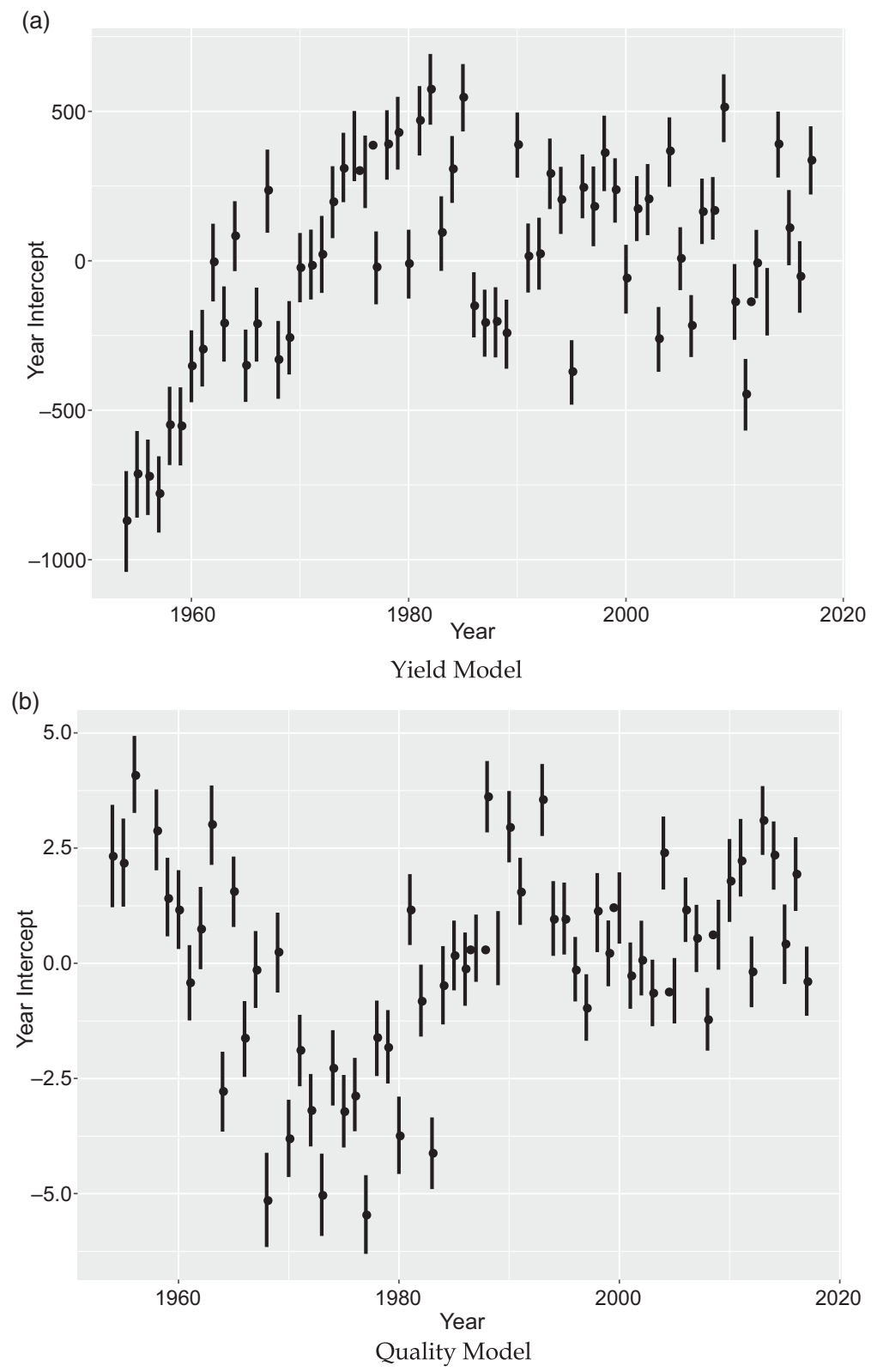

Figure 3. Time intercepts: technology and management practice contributions.

Note: Time intercept in the yield model is interpreted as lbs./acre relative to the average yield given by $\mu$. Time intercept in the quality model is interpreted as sugars/alkaloids ratio relative to the average quality given by $\mu$.

$$
A G C_{t}=\left(\frac{1}{L}\right) \sum_{i=1}^{L} a_{i[t]}
$$

where $L$ is the number of varieties introduced in year $t$ and $\alpha_{i[t]}$ denotes the varying intercept of variety $i$ if introduced in year $t$. If interested in the contribution to yields, then the averaged variety intercepts are from the yield equation, and similarly for quality measures. Breeding efforts can vary from year to year and it may be more natural to consider average genetic improvement 


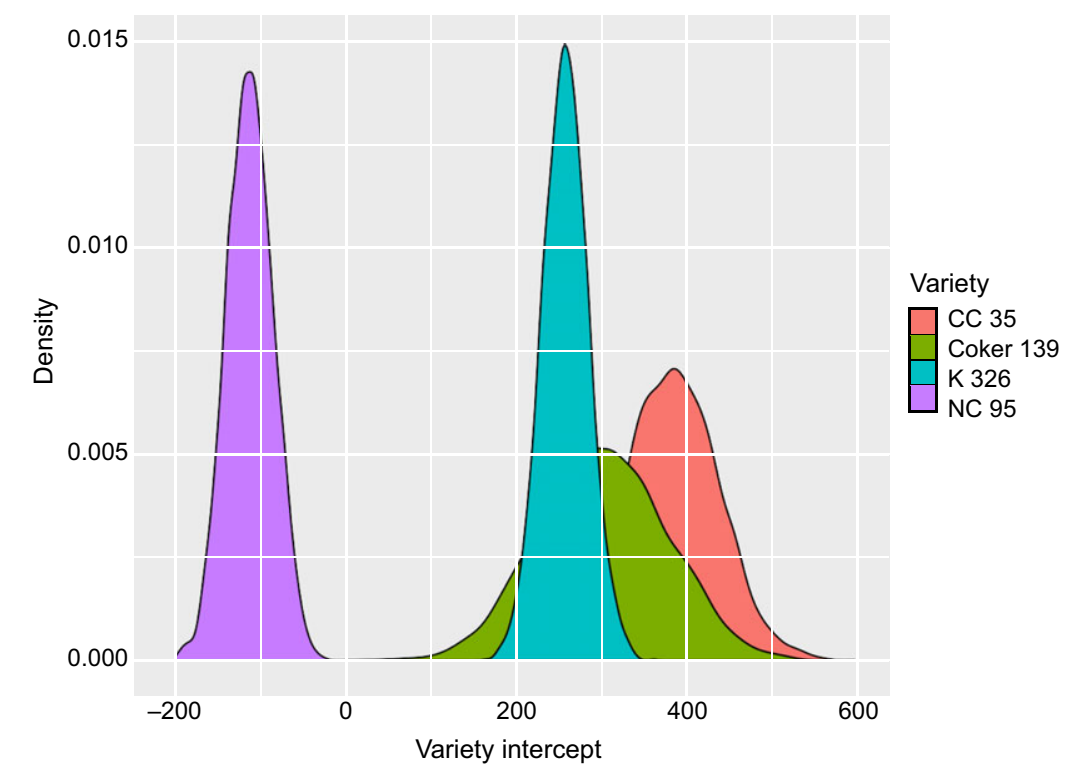

Figure 4. Posterior density of selected variety intercepts for yield.

Note: Variety intercept in the yield model is interpreted as lbs./acre relative to the average yield given by $\mu$.

in a given year. Though increased overall efforts in a given year (e.g., breeding and releasing a large number of new varieties) do not automatically enhance breeding-based gains in yields, more breeding effort may increase the likelihood of having several high-yield varieties with the best quality traits. This can consequently push up the overall mean yield for all released varieties.

Another quantity of interest is the maximum genetic contribution across all varieties introduced in that year. The formula would be

$$
\operatorname{MGC}_{t}=\max \left(\alpha_{i[t]}\right)
$$

and more clearly represents the best variety in a given year. The maximum genetic contribution (MGC) recognizes the uncertainty that surrounds research and development. Most varieties introduced may not be meaningful in terms of their contribution to yields or quality (e.g., not all varieties released in a year will be "hits" with the highest yields and the best quality). While it would be trivial to construct a point estimate of AGC or MGC in a frequentist framework, it would not be trivial to generate estimates of uncertainty surrounding this quantity in a frequentist context. In the Bayesian paradigm, on the other hand, we confront no such challenge as the uncertainty of any function of the parameters is directly ascertained through the draws from the posterior distribution.

Figure 5 shows the average genetic contribution (AGC) and MGC plotted over time with the dots again representing posterior medians and the bars giving $95 \%$ posterior credible intervals. AGC increases over time with considerable uncertainty in the estimates. This should be expected given that we are averaging over all varieties introduced in a particular year. Nonetheless, the modest increases observed in the AGC plot suggest that overall breeding efforts over time have resulted in flue-cured tobacco varieties that on average have higher yields (e.g., the mean yield effects are still positive on average even when aggregating the "non-hit" varieties with the "hit" varieties released over time). The estimates based on MGC also show increased varietal contribution to yield, but with a more positive upward slope especially after the 1980s. The breedingbased yield contributions of the discount varieties in 1954 (which constitute MGC in 1954) are roughly equivalent to the best modern varieties, but the best modern varieties have more favorable 

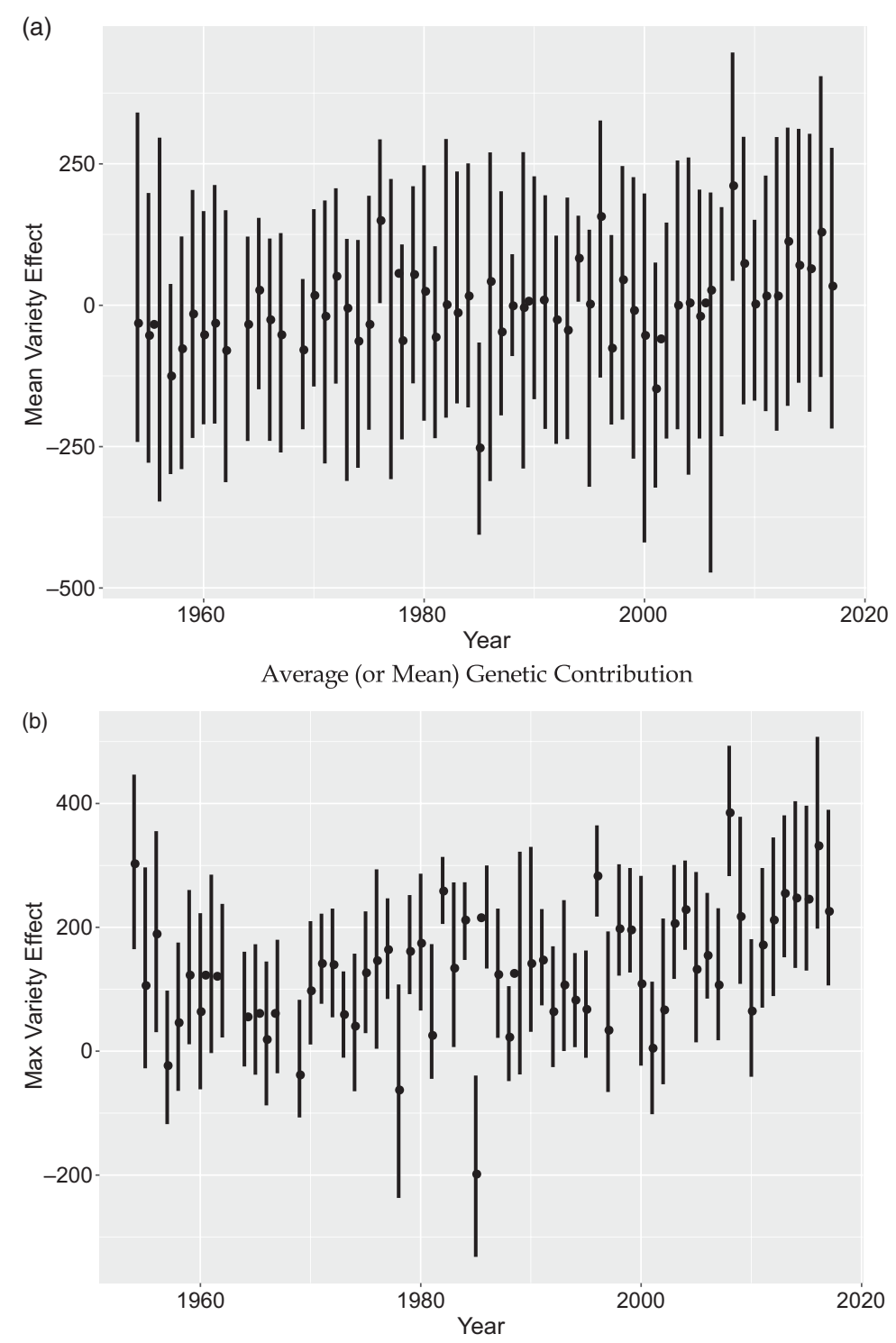

Maximum Genetic Contribution

Figure 5. Genetic contribution by year.

Note: Variety intercept in the yield model is interpreted as lbs./acre relative to the average yield given by $\mu$.

quality characteristics. Hence, the best breeding outcomes have generated notable breeding-based yield gains without compromising tobacco quality attributes.

The standard deviations for the location, time, and variety intercepts give an idea of the variation in yields that can be attributed to each factor. Trace plots of the standard deviations, as well as posterior densities, are shown in Figure 6 . In terms of the variances, the year intercepts capture the most variance, followed by the location intercepts and the variety intercepts. The standard deviation for the variety intercepts is roughly half that of the year intercepts indicating that more of the variation is captured by changes across years than across varieties. Note that there is also variation across locations, but the distribution is wide, indicating a high degree of uncertainty. 

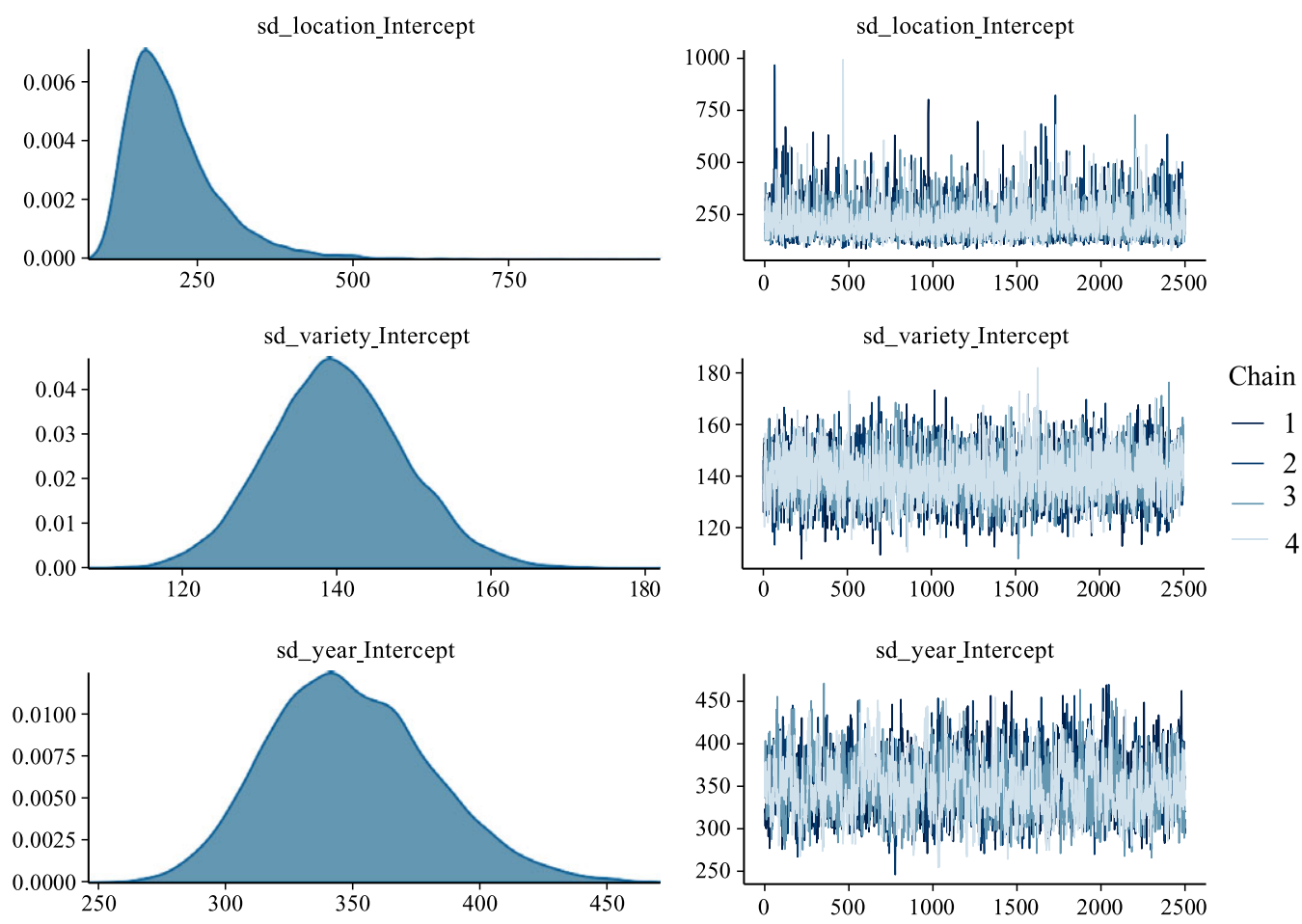

Figure 6. Standard deviation parameters.

The relative contributions of varietal improvement (relative to technology) can be obtained from changes in the variety and time-varying intercepts. Based on plots of yields in Figure 1, we divide the sample into three periods 1963-1981, 1981-2000, and 2000-2017. We then calculate the change in breeding contribution (the variety-specific intercept) over this period. The way that breeding-based yield contribution is defined is important for the results. Babcock and Foster (1991) measure the contribution as the difference between the two slope coefficients associated with older, standard varieties versus new varieties; both are estimated in a frequentist framework. In order to derive the measure of varietal contribution, they impose a strict model of how varietal improvement occurs as well as being forced to group varieties into two distinct categories.

Because our underlying model is more flexible, we can measure varietal improvement in several ways. The chosen approach requires a choice of when and how to measure. For the results that follow, we use AGC and MGC to measure the relative contribution of tobacco breeding, and then use the year intercepts to calculate the relative contribution of improved management practices and/or production technology aside from breeding-based advances (over the same time period). For instance, to measure the change in yields attributable to a variety from 1963 to 1981, we calculate the average MGC over 1980, 1981, and 1982, and then subtract the average MGC over 1962, 1963, and 1964. A similar approach is used to measure the change in yields attributable to improved management practices and production technology (excluding varietal improvement), but using the year intercepts. It is important to note that we only use varieties introduced in the 3 years at the beginning and end of each of the periods.

Table 2 contains the results of these calculations for yields. A similar table of results for quality is given in Table A1 of the appendix; the changes in quality are not substantial with the ratio remaining within the acceptable range. This approach essentially ignores varieties released in intervening years. This may account for the negative estimates between 1981 and 2000 . 
Table 2. Measured contribution of genetics to changes in yield

\begin{tabular}{lccc}
\hline Period & Genetics & Technology & Relative Contribution (\%) \\
\hline AGC & & & \\
\hline $1963-1981$ & 38.50 & 387.74 & 9.03 \\
\hline $1981-2000$ & -49.75 & -225.66 & 18.06 \\
\hline $2000-2017$ & 144.50 & 12.58 & 91.99 \\
\hline MGC & & & 11.80 \\
\hline $1963-1981$ & 51.87 & 387.74 & 17.87 \\
\hline $1981-2000$ & -49.09 & -225.66 & 92.91 \\
\hline $2000-2017$ & 164.73 & 12.58 & 9.06 \\
\hline
\end{tabular}

Note: Columns 1 and 2 denote pound per acre changes in average genetic contribution or maximum genetic contribution between varieties introduced at the beginning and end of the time periods. Column 3 measures relative contribution of genetics as percentage of change due to genetics and technology.

Simply put, the varieties introduced in 1980-1982 had greater varietal and technological contribution to yield relative to those varieties introduced from 1999 to 2001 . Varieties introduced in 2000 and 2001 had low variety-specific intercepts. The research process does not deliver constant increases in yield or quality every year, but rather involves experimentation to develop improved varieties. Therefore, some years may not involve any new varieties with improved yield or quality. Another explanation for the decline between 1981 and 2002 may be that changes in tobacco policy resulted in changes to management practices.

Our two salient findings are as follows. First, both varietal improvement and technology contributed to yield gains before 1981, but the contribution of technology is much larger in this earlier period. Second, in the last period (2000-2017), there was little contribution from technology to improved yields, but there were significant advancements in terms of breeding. This conclusion is consistent with estimated break points in a piecewise regression of variety intercepts on time. These conclusions are also consistent with previous results including those for specific varieties shown in Figure 4.

Our results above suggest that the contribution of varietal improvement and non-breedingbased technological change to revenue of the tobacco sector has been substantial. The price of flue-cured tobacco in 2019 was $\$ 1.88$ per pound. Using the posterior median of the difference between the variety intercept of NC 95 (i.e., an old variety) and K 326 (i.e., a new variety), a field planted to NC 95 would return $\$ 692.63$ less per acre relative to K326. A similar calculation using the difference between K 326 and CC 35 (i.e., a new higher-yielding variety) shows that a field planted to CC 35 would return $\$ 232.94$ more per acre as compared to K 326 . If we use CCC discount rates for low-quality tobacco, Coker 139 has a breeding-based contribution of roughly 302 pounds to yield, but receives only $\$ 0.94$ per pound due to low quality. Total revenue is still positive regardless of the price discount but substantially less than revenue received under $\mathrm{K} 326$ by a factor of roughly $50 \%$. The previous calculations ignore any changes to costs that might occur between varieties. Given increases in wage rates for agricultural labor and other inputs, public and private research in tobacco has enabled flue-cured farmers to maintain positive net revenues and ensured the viability of the industry.

Hedonic analyses or price information from quality-differentiated tobacco sales would allow us to more accurately distinguish the contribution of quality to revenue. However, with the end of the tobacco quota program, producers no longer have an incentive to produce varieties with high yields and low quality. The vast majority of production is now contracted and some production contracts may specify the variety of tobacco that is to be grown. More generally, contracts provide a means for buyers to obtain differentiated products and guarantee certain methods of production 

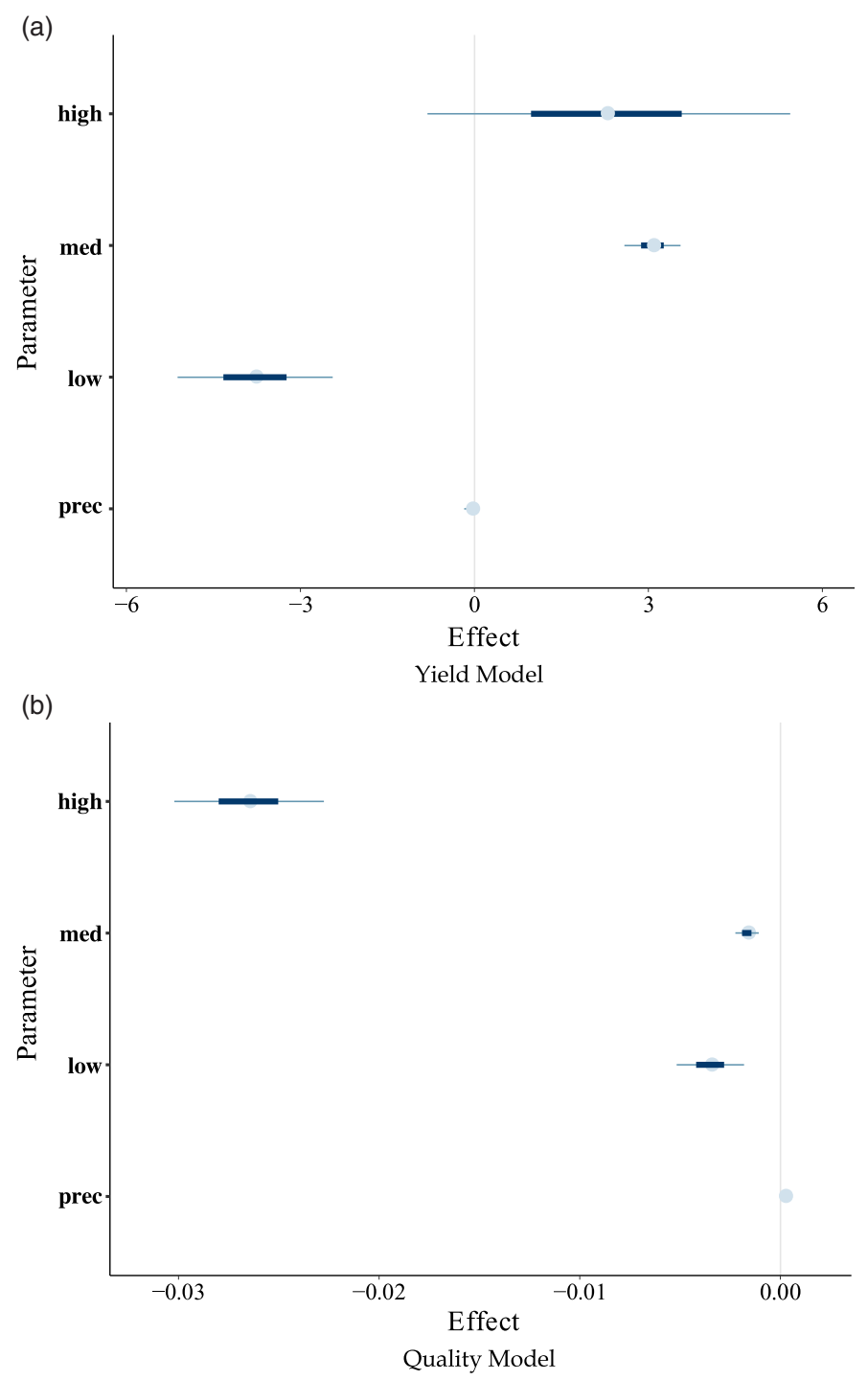

Figure 7. Climate function coefficients.

(MacDonald et al., 2004). While quality is still a determinant of tobacco revenue, our ability to determine the precise extent to which quality affects revenue is hidden behind the current production and marketing systems in tobacco markets.

Although not the main variables of interest in this study, we also examined the effects of the weather variables on yield and quality, and the results are shown in Figure 7. The resilience of tobacco yields to high heat is evident. Both medium and high temperatures contribute to increased yields although there is some uncertainty surrounding the positive estimate for high temperatures. In contrast, low temperatures have a large negative effect on yields. Although the posterior median of the coefficient on precipitation is negative, there is also uncertainty as the $95 \%$ credible interval is $(-0.2,0.09)$. Being grown under best management practices, tobacco in the variety trials was likely irrigated in periods of low precipitation. The use of irrigation to counteract drought may result in the uncertainty around the precipitation parameter. We find an asymmetric effect of high 
temperatures on yield and quality that is consistent with the type of tradeoff suggested in Kawasaki and Uchida (2016) and contrasts with symmetric effects reported in Ramsey et al. (2021). All temperatures have negative effects on quality, with the most serious for high-temperature degree days.

\section{Conclusions}

We examine the contribution of breeding and other technologies to changes in yield and quality of flue-cured tobacco from 1954 to 2017. Both varietal improvement and other technological change contributed to yield increases up until 1980 at which point yield potential reached a plateau or possibly declined. After 2000, yields began to see renewed improvement from breeding advances. No appreciable changes occurred in quality given the voluntary quality standards agreed to by the industry. Results indicate that it is possible to make varietal improvements in terms of yield while maintaining minimum quality standards. Breeding trade-offs between yields and quality levels were not evident in the flue-cured tobacco OVT data in recent years. Breeders successfully targeted several aspects of the crop, from yield potential to quality, with consequent impacts on revenue received by producers.

Our empirical findings suggest that investments in public and private tobacco breeding programs have resulted in improved flue-cured tobacco varieties and enhanced yield potential. However, without information on funding toward tobacco research, we cannot make any definitive conclusions as to changes in net return on investment. The voluntary quality standards in tobacco did not serve as a barrier to generating further yield gains from breeding. In terms of policy implications, there is no need to relax industry quality standards in order to achieve yield gains from varietal improvement. Continued investents in conventional breeding to achieve higher yields may be warranted even with the current regulatory structure on quality.

It is important to note that none of the varieties of tobacco included in this study are transgenic. We can reasonably assume that if the market is willing to accept transgenic tobacco, higheryielding varieties of high quality might be developed in the future through transgenic breeding. Several studies with transgenic plants have shown better leaf mass, resistance to environmental stresses, and better nutrient uptake (Tarczynski et al., 1993; Lopez-Bucio et al., 2000). Advances in transgenic breeding for tobacco may lead to greater yield potential, which is one route to improved farm revenues in the face of tightening margins. Nonetheless, whether quality-and farm revenue - could be maintained through transgenic modification remains an open question.

Although our study provides insight into the economics of tobacco breeding and introduces a method for understanding yield and quality tradeoffs in breeding programs, it also raises several limitations and promising future research directions. First, the OVT data in the study do not include detailed information on management practices and specific genetic traits of each variety. If we observe location-specific management and genetic-trait variables, these could be included in the group-level model for the location-varying intercepts. Tack et al. (2017) contains a frequentist example of this type of hierarchical modeling approach. More detailed information on planting and harvest dates would also allow improved specification of the climate function. If available, additional information about variety-specific genetic traits can also serve as additional controls in the specification, which would help in more accurately estimating specific genetic trait effects. Second, our study primarily focused on examining the mean yield and mean quality effects of varietal improvement. It might also be useful to extend the model beyond the mean as in Tack et al. (2012) and Zhu et al. (2011) and investigate some of the risk implications of crop breeding. Lastly, because yield and quality measures were not available in all years, the econometric models in our study are estimated independent of one another. While possible to model correlation in the error term or coefficients, little might be gained from doing so given that both 
equations contain the same independent variables (i.e., parameter estimates would be similar, regardless if both equations are estimated separately or jointly).

Acknowledgements. This research would not have been possible without generous assistance from Matthew Vann, Ramsey Lewis, and William Foote of the NC State University Department of Crop and Soil Sciences and NC Crop Improvement Association. The helpful comments of seminar participants at the 49th Tobacco Workers' Conference and the University of Kentucky are gratefully acknowledged.

Supplementary material. For supplementary material accompanying this paper visit https://doi.org/10.1017/aae.2021.25

Financial Support. This research received no specific grant from any funding agency, commercial or not-for-profit sectors.

Conflict of Interest. A. Ford Ramsey and Roderick M. Rejesus declare none.

Data Statement. The data that support the findings of this study are available from A. Ford Ramsey. Data are available from the authors with the permission of the tobacco variety testing program at North Carolina State University.

\section{References}

Babcock, B.A., and W.E. Foster. "Measuring the Potential Contribution of Plant Breeding to Crop Yields: Flue-cured Tobacco, 1954-87." American Journal of Agricultural Economics 73,3(1991):850-59.

Betancourt, M.J., and M. Girolami. "Hamiltonian Monte Carlo for Hierarchical Models." Preprint, arXiv:1312.0906 [stat.ME], 2013.

Bowman, D. "History of the Regional Minimum Standards Program for the Release of Flue-cured Tobacco Varieties in the United States." Tobacco Science 40(1996):99-110.

Bowman, D., E. Wernsman, T. Corbin, and A. Tart. "Contribution of Genetics and Production Technology to Long-Term Yield and Quality Gains in Flue-Cured Tobacco.” Tobacco Science 28(1984):30-35.

Brink, L., and B. McCarl. "The Tradeoff between Expected Return and Risk among Cornbelt Farmers." American Journal of Agricultural Economics 60,2(1978):259-63.

Brown, A.B., R.R. Rucker, and W.N. Thurman. "The End of the Federal Tobacco Program: Economic Impacts of the Deregulation of us Tobacco Production." Review of Agricultural Economics 29,4(2007):635-55.

Bruckner, H. The Chemical Determination of Tobacco Quality. Berlin: Paul Parey, 1936.

Bürkner, P.C., et al. "brms: An R Package for Bayesian Multilevel Models using Stan.” Journal of Statistical Software 80,1(2017):1-28.

Chavas, J.P., K. Kim, J.G. Lauer, R.M. Klemme, and W.L. Bland. “An Economic Analysis of Corn Yield, Corn Profitability, and Risk at the Edge of the Corn Belt." Journal of Agricultural and Resource Economics 26(2001):230-47.

Dimitri, C. Contracting in Tobacco?: Contracts Revisited. Washington, DC: US Department of Agriculture, Economic Research Service, 2003.

Donnet, M.L., D.D. Weatherspoon, and C.B. Moss. "Measuring Food Product Differentiation by Quality Ratings: A CrossEntropy Analysis of Specialty Coffee e-Auctions." Journal of Agricultural Economics 61,1(2010):122-37.

Espinosa, J.A., and B.K. Goodwin. "Hedonic Price Estimation for Kansas Wheat Characteristics." Western Journal of Agricultural Economics 16(1991):72-85.

Gabry, J., D. Simpson, A. Vehtari, M. Betancourt, and A. Gelman. "Visualization in Bayesian Workflow." Journal of the Royal Statistical Society: Series A (Statistics in Society) 182,2(2019):389-402.

Gelman, A. "Analysis of Variance-Why It Is More Important than Ever." The Annals of Statistics 33,1(2005):1-53.

Gelman, A., and J. Hill. Data Analysis using Regression and Multilevel/Hierarchical Models. Cambridge: Cambridge University Press, 2006.

Golan, A., and H. Shalit. "Wine Quality Differentials in Hedonic Grape Pricing." Journal of Agricultural Economics 44,2(1993):311-21.

Greif, A. "Contract Enforceability and Economic Institutions in Early Trade: The Maghribi Traders' Coalition.” American Economic Review 83(1993):525-48.

Hawks, S.N., and W.K. Collins. Principles of Flue-Cured Tobacco Production. Raleigh, NC: Collins and Hawks, 1993.

Hussein, M., and I. Fraser. "Hedonic Analysis of Consumers' Valuation of Country of Origin of Meat in the United Kingdom." Journal of Agricultural Economics 69,1(2018):182-98.

Kawasaki, K. "Two Harvests are Better than One: Double Cropping as a Strategy for Climate Change Adaptation.” American Journal of Agricultural Economics 101,1(2018):172-92.

Kawasaki, K., and E. Lichtenberg. "Econometric Analysis of Grading Standards: The Ordered Fractional Approach." American Journal of Agricultural Economics 96,1(2013):345-65.

Kawasaki, K., and S. Uchida. "Quality Matters More than Quantity: Asymmetric Temperature Effects on Crop Yield and Quality Grade.” American Journal of Agricultural Economics 98,4(2016):1195-209. 
Lobell, D.B., W. Schlenker, and J. Costa-Roberts. “Climate Trends and Global Crop Production Since 1980.” Science 333,6042(2011):616-20.

Lopez-Bucio, J., O.M. de la Vega, A. Guevara-Garcia, and L. Herrera-Estrella. "Enhanced Phosphorus Uptake in Transgenic Tobacco Plants that Overproduce Citrate.” Nature Biotechnology 18,4(2000):450-53.

MacDonald, J.M., J. Perry, M.C. Ahearn, D. Banker, W. Chambers, C. Dimitri, N. Key, K. E. Nelson, and L.W. Southard. "Contracts, Markets, and Prices: Organizing the Production and Use of Agricultural Commodities." USDA-ERS Agricultural Economic Report 837, 2004.

MacLeod, W.B. “Reputations, Relationships, and Contract Enforcement." Journal of Economic Literature 45,3(2007): 595-628.

Maligalig, R., M. Demont, W.J. Umberger, and A. Peralta. "Understanding Filipino Rice Farmer Preference Heterogeneity for Varietal Trait Improvements: A Latent Class Analysis." Journal of Agricultural Economics 72,1(2021):134-57.

Maw, B.W., J.R. Stansell, and B.G. Mullinix. "Soil-Plant-Water Relationships for Flue-Cured Tobacco." UGA Cooperative Extension Bulletin 427(2009):1-36.

Muggeo, V.M. “Segmented: An R Package to Fit Regression Models with Broken- Line Relationships." R News 8,1(2008): $20-25$.

Nalley, L.L., A.P. Barkley, and F.G. Chumley. "The Impact of the Kansas Wheat Breeding Program on Wheat Yields, 1911-2006." Journal of Agricultural and Applied Economics 40,1379-2016-112782(2008):913-25.

Nalley, L.L., A.P. Barkley, and A.M. Featherstone. "The Genetic and Economic Impact of the Cimmyt Wheat Breeding Program on Local Producers in the Yaqui Valley, Sonora Mexico." Agricultural Economics 41,5(2010):453-62.

Nalley, L., B. Dixon, P. Chaminuka, Z. Naledzani, and M. J. Coale. The Role of Public Wheat Breeding in Reducing Food Insecurity in South Africa." PloS One 13,12(2018):1-20.

Neal, R. "Mcmc using Hamiltonian Dynamics." Handbook of Markov Chain Monte Carlo. S. Brooks, A. Gelman, G. Jones, and X.L. Meng, eds. Boca Raton: Chapman and Hall/CRC, 2011, pp. 116-62.

Nogueira, L., J. Michalski, T.L. Marsh, and V. McCracken. "Welfare Implications of Washington Wheat Breeding Programs." Journal of Agricultural and Applied Economics 47,2(2015):147-74.

Nolan, E., and P. Santos. "The Contribution of Genetic Modification to Changes in Corn Yield in the United States." American Journal of Agricultural Economics 94,5(2012):1171-88.

Pyriki, C. "Relations between the Chemical Composition of Tobacco and the Characteristics of Smoke." Proceedings of the 2nd International Tobacco Science Congress (1958):460-95.

Ramsey A.F., J. B. Tack, and M. Balota. "Double or Nothing: Impacts of Warming on Crop Quantity, Quality, and Revenue." Journal of Agricultural and Resource Economics (2021):Forthcoming.

Rucker, R.R., W.N. Thurman, and D.A. Sumner. "Restricting the Market for Quota: An Analysis of Tobacco Production Rights with Corroboration from Congressional Testimony.” Journal of Political Economy 103,1(1995):142-75.

Schlenker, W., and M.J. Roberts. "Nonlinear Effects of Weather on Corn Yields." Review of Agricultural Economics 28,3(2006):391-98.

Schlenker, W., and M.J. Roberts. "Nonlinear Temperature Effects Indicate Severe Damages to us Crop Yields under Climate Change." Proceedings of the National Academy of Sciences 106,37(2009):15594-98.

Shew, A.M., A. Durand-Morat, L.L. Nalley, and K.A.K. Moldenhauer. "Estimating the Benefits of Public Plant Breeding: Beyond Profits." Agricultural Economics 49,6(2018):753-64.

Shi, G., J.P. Chavas, and J. Lauer. "Commercialized Transgenic Traits, Maize Productivity and Yield Risk." Nature Biotechnology 31,2(2013a):111-14.

Shi, G., J.P. Chavas, J. Lauer, and E. Nolan. "An Analysis of Selectivity in the Productivity Evaluation of Biotechnology: An Application to Corn.” American Journal of Agricultural Economics 95(3) (2013b):739-54.

Stiegert, K., and J.P. Blanc. "Japanese Demand for Wheat Protein Quantity and Quality." Journal of Agricultural and Resource Economics 22(1997):104-119.

Tack, J., A. Barkley, and L. Lanier Nalley. "Estimating Yield Gaps with Limited Data: An Application to United States Wheat. American Journal of Agricultural Economics 97,5(2015):1464-77.

Tack, J., A. Harri, and K. Coble. "More than Mean Effects: Modeling the Effect of Climate on the Higher Order Moments of Crop Yields. American Journal of Agricultural Economics 94,5(2012):1037-54.

Tack, J., J. Lingenfelser, and S.K. Jagadish. "Disaggregating Sorghum Yield Reductions under Warming Scenarios Exposes Narrow Genetic Diversity in us Breeding Programs." Proceedings of the National Academy of Sciences 114,35(2017): 9296-301.

Tarczynski, M.C., R.G. Jensen, and H. J. Bohnert. "Stress Protection of Transgenic Tobacco by Production of the Osmolyte Mannitol." Science 259(5094) (1993):508-10.

Tso, T.C. Physiology and Biochemistry of Tobacco Plants. Stroudsburg, PA: Dowden, Hutchinson \& Ross, Inc., 1972.

Voon, J.P., and G.W. Edwards. "Research Payoff from Quality Improvement: The Case of Backfat Depth in Pigs." Journal of Agricultural Economics 42,1(1991):66-76.

Voon, T.J., and G.W. Edwards. "Research Payoff from Quality Improvement: The Case of Protein in Australian Wheat." American Journal of Agricultural Economics 74,3(1992):564-72. 
Wang, R., R.M. Rejesus, J. B. Tack, J. V. Balagtas, and A. D. Nelson. "Quantifying the Yield Sensitivity of Modern Rice Varieties to Warming Temperatures: Evidence from the Philippines." American Journal of Agricultural Economics (2021). doi: 10.1111/ajae.12210.

Weybrew, J., W. Wan Ismail, and R. Long. "The Cultural Management of Flue-Cured Tobacco Quality." Tobacco International 185,10(1983):82-87.

Zhu, Y., B.K. Goodwin, and S.K. Ghosh. "Modeling Yield Risk under Technological Change: Dynamic Yield Distributions and the US Crop Insurance Program." Journal of Agricultural and Resource Economics 26(2011):192-210.

\section{Appendix}

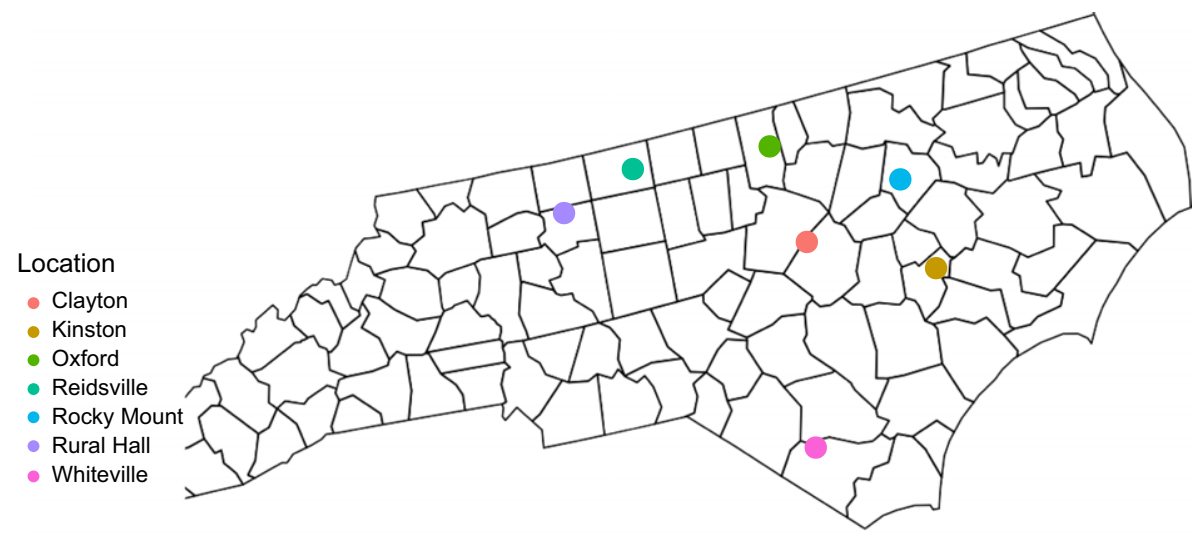

Figure A1. Map of trial locations.

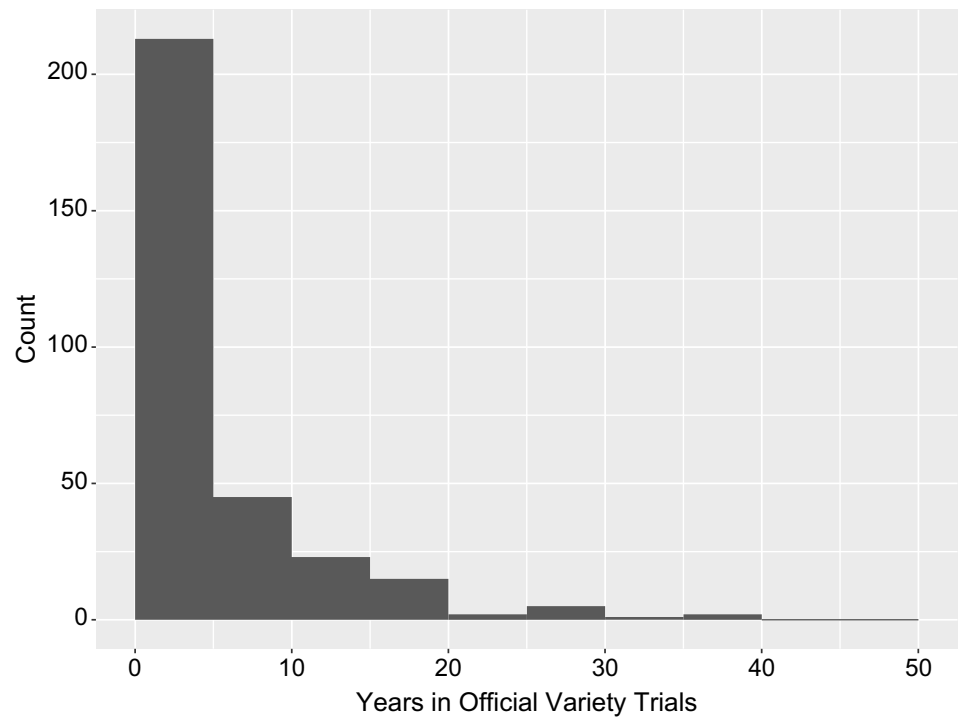

Figure A2. Histogram of appearances in Official Variety Trials (OVT) by variety. 
(a)

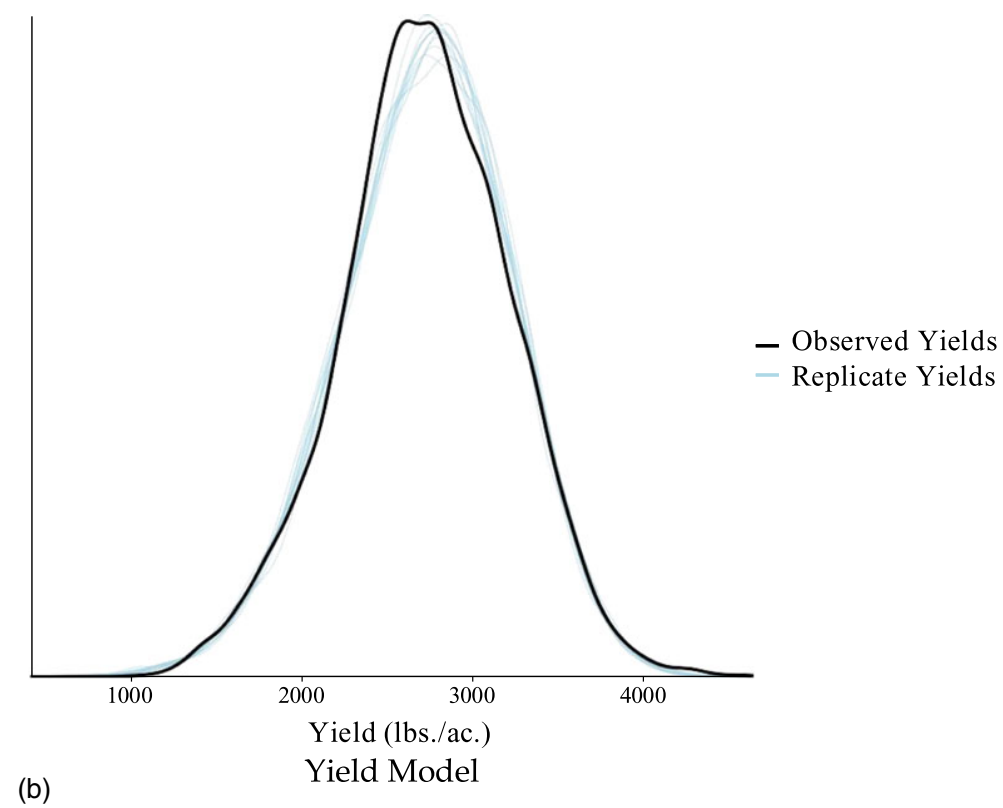

(b)

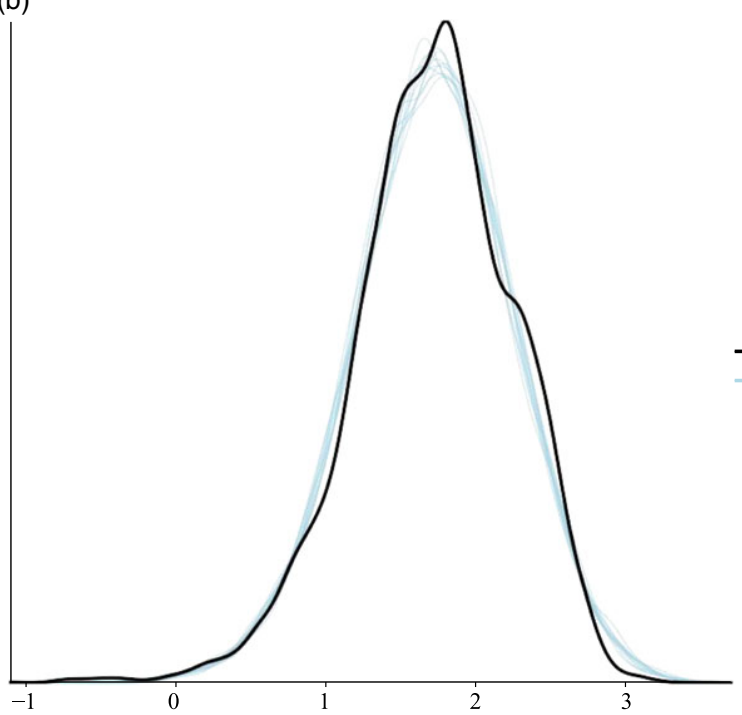

$\log$ (Sugars/Alkaloids)

Quality Model

Figure A3. Posterior predictive checks.

Note: Replicates are drawn using the same data used to fit the models. 
Table A1. Measured contribution of genetics to change in quality

\begin{tabular}{|c|c|c|c|}
\hline Period & Genetics & Technology & Relative Contribution \\
\hline \multicolumn{4}{|l|}{ AGC } \\
\hline 1963-1981 & 0.02 & -0.31 & $6.06 \%$ \\
\hline 1981-2000 & -0.02 & 0.32 & $5.88 \%$ \\
\hline 2000-2017 & 0.01 & 0.07 & $12.5 \%$ \\
\hline \multicolumn{4}{|l|}{ MGC } \\
\hline 1963-1981 & 0.03 & -0.31 & $8.82 \%$ \\
\hline $1981-2000$ & -0.03 & 0.32 & $8.57 \%$ \\
\hline 2000-2017 & 0.04 & 0.07 & $36.36 \%$ \\
\hline
\end{tabular}

Note: Columns 1 and 2 denote sugars/alkaloids ratio changes in average genetic contribution or maximum genetic contribution between varieties introduced at the beginning and end of the time periods. Column 3 measures relative contribution of genetics as percentage of change due to genetics and technology.
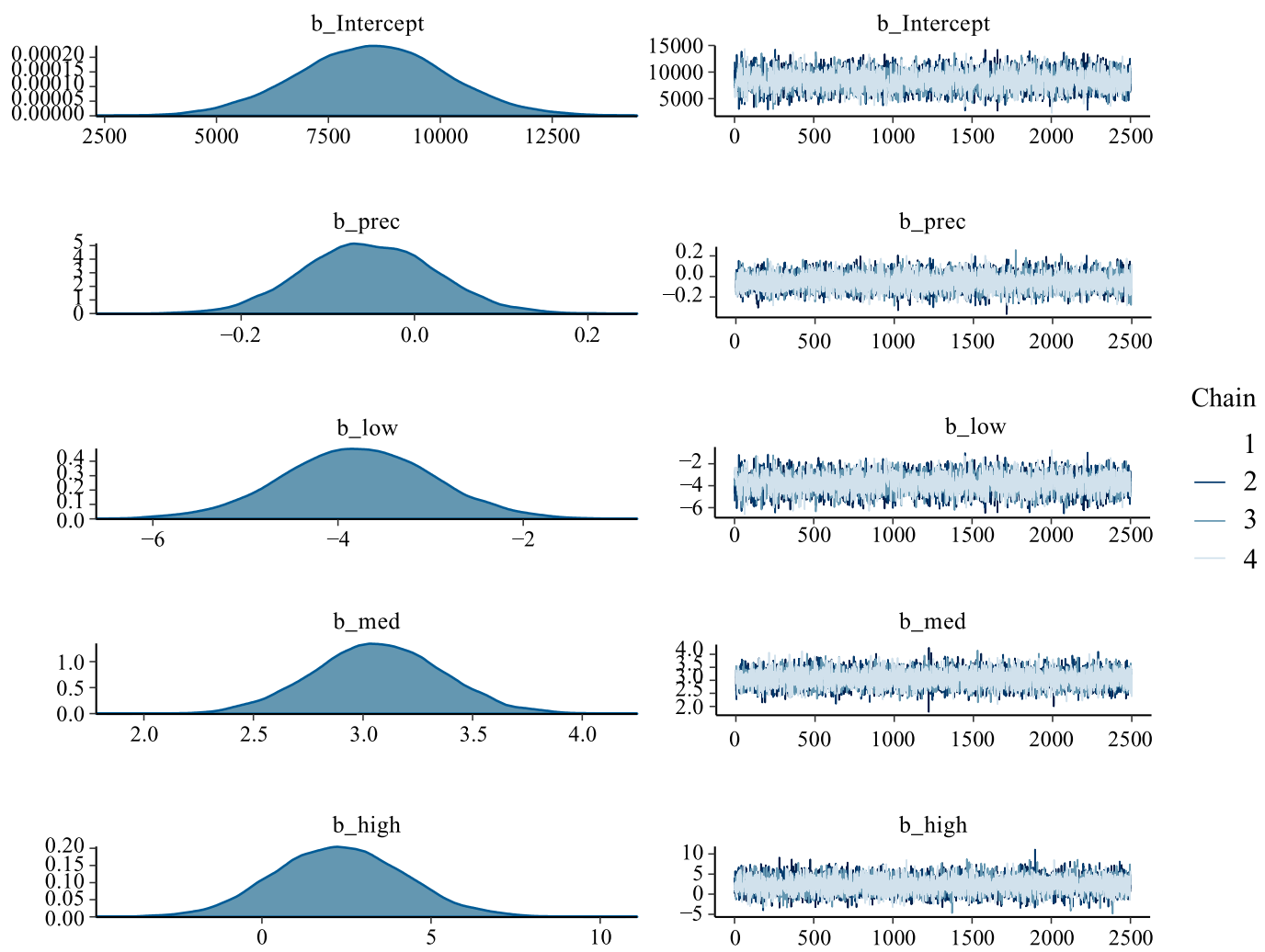

Figure A4. Trace plots of selected yield model parameters.

Cite this article: Ramsey, A.F. and R.M. Rejesus. "Bayesian Hierarchical Models for Measuring Varietal Improvement in Tobacco Yield and Quality." Journal of Agricultural and Applied Economics (2021), 53, 563-586. https://doi.org/10.1017/aae.2021.25 